Journal for ImmunoTherapy of Cancer

\section{Immunogenicity in humans of a transdermal multipeptide melanoma vaccine administered with or without a TLR7 agonist}

To cite: Meneveau M0, Petroni GR, Salerno EP, et al. Immunogenicity in humans of a transdermal multipeptide melanoma vaccine administered with or without a TLR7 agonist. Journal for ImmunoTherapy of Cancer 2021;9:e002214. doi:10.1136/jitc-2020-002214

- Additional material is published online only. To view, please visit the journal online (http://dx.doi.org/10.1136/jitc2020-002214).

Accepted 01 March 2021

Check for updates

(C) Author(s) (or their employer(s)) 2021. Re-use permitted under CC BY-NC. No commercial re-use. See rights and permissions. Published by BMJ.

${ }^{1}$ Surgery, University of Virginia School of Medicine, Charlottesville, Virginia, USA ${ }^{2}$ Public Health Sciences, University of Virginia School of Medicine, Charlottesville, Virginia, USA

${ }^{3}$ Cancer Center, University of Virginia Health System, Charlottesville, Virginia, USA ${ }^{4}$ Pathology, University of Virginia, Charlottesville, Virginia, USA ${ }^{5}$ Medicine, University of Virginia School of Medicine, Charlottesville, Virginia, USA

Correspondence to

Dr Craig L Slingluff;

cls8h@virginia.edu

\section{ABSTRACT}

Background Experimental cancer vaccines are traditionally administered by injection in subcutaneous tissue or muscle, commonly with adjuvants that create chronic inflammatory depots. Injection of melanomaderived peptides induces T cell responses; however, the depots that form following injection may inhibit optimization of the immune response. In skin, epidermal Langerhans cells (LC) are a dominant source of professional antigen presenting cells. We hypothesized that: (1) applying melanoma-derived peptides topically, in proximity to LC, could be immunogenic and safe, with low vaccine-site toxicity and (2) topical toll-like receptor 7 (TLR7) agonist would increase immunogenicity of the peptide vaccine.

Methods Twelve melanoma peptides plus a tetanus helper peptide were combined with granulocyte macrophage colony stimulating factor (GM-CSF) and were administered topically on days 1,8 , and 15 , to 28 patients randomized to one of four adjuvant preparations: (1) incomplete Freund's adjuvant (IFA); (2) IFA plus a TLR7 agonist (imiquimod) administered on days 0, 7, 14; (3) dimethyl sulfoxide (DMSO) or (4) DMSO+ imiquimod administered on day $0,7,14$. Every 3 weeks thereafter $(x$ $6)$, the peptides were combined with GM-CSF and were injected into the dermis and subcutis in an emulsion with IFA. Toxicities were recorded and immune responses assayed by ELIspot.

Results $\mathrm{CD}^{+} \mathrm{T}$ cell responses to transdermal vaccination in DMSO occurred in $83 \%$ of participants in group 3 and $86 \%$ in group 4 , and responses to vaccination in IFA were observed in $29 \%$ of participants in group 1 and $14 \%$ in group 2. Overall, $61 \%$ of participants had CD4 ${ }^{+}$ $T$ cell immune responses to the tetanus peptide, with large, durable responses in groups 3 and 4 . Five of seven participants in group 4 had a severe rash, one that was dose limiting. Ten-year overall survival was $67 \%$ and disease-free survival was $44 \%$.

Conclusions These data provide proof of principle for immunogenicity in humans of transdermal immunization using peptides in DMSO. Further study is warranted into the pharmacokinetics and immunobiology of TLR agonists as vaccine adjuvants during transcutaneous application.
Overall survival is high, supporting further investigation of this immunization approach.

\section{INTRODUCTION}

Cell-based antitumor vaccines employing reintroduction of dendritic cells (DCs) after ex vivo manipulation have been shown to be safe and effective in some melanoma patients. ${ }^{1-5}$ DCs have potent antigen presenting cell properties, making them appealing components of antitumor immune therapy. However, the requisite time, effort, cost and lack of benefit in most clinical trials of ex vivo culture and adoptive transfer of DC vaccines limits their broad applicability. ${ }^{46-8}$ Alternatively, DCs may be activated in situ in the skin when vaccines are administered in the skin. Langerhans cells (LCs) are DCs residing in the epidermis that, when matured, preferentially stimulate naïve $\mathrm{T}$ cell differentiation into cytotoxic effector cells. Subcutaneous or intramuscular vaccination with peptides in adjuvant has been more immunogenic than vaccination with peptide-pulsed $\mathrm{DCs}^{4}{ }^{9}$; however, injection of vaccines into subcutaneous or intramuscular locations places the antigens at some distance from LCs. Additionally, peptide antigens are subject to rapid degradation by proteolytic enzymes in the skin. ${ }^{10}$ In contrast, peptides applied topically on the skin have significantly longer half-lives and are anatomically much closer to the epidermal LCs than those administered subcutaneously or even intradermally. ${ }^{12}$ We have hypothesized that a transdermal delivery system for a peptidebased vaccine may be effective for inducing melanoma reactive $\mathrm{T}$ cells.

The prospect of a non-invasive, simple and economic method of immunization has been the driving force for development of 
transcutaneous immunization. ${ }^{13-17}$ In preclinical models, transdermal vaccines have induced protective immunity and antitumor T cell responses. ${ }^{18-20}$ Transdermal immunization can also generate antibody responses to topically applied cholera toxin, diphtheria toxoid and tetanus toxoid. ${ }^{21}$ The humoral and cellular responses to antigens administered in these studies suggest that transdermal immunization may be a valid method for cancer immunotherapy. In a murine model of melanoma, topically administered small synthetic melanoma peptides mixed in imiquimod cream (a pharmaceutical toll-like receptors 7 (TLR7) agonist) induced strong cytotoxic T-lymphocyte (CTL) responses and improved overall survival of tumor-bearing mice. ${ }^{22} \mathrm{~A}$ more recent murine study has demonstrated potent immunogenicity and lasting antitumor activity with transcutaneous vaccination using synthetic peptide combined with CTLA-4 blockade..$^{20}$ In clinical studies, stripping the stratum corneum prior to transdermal peptide vaccination has shown promise. ${ }^{23}$

The function of vaccine adjuvants is not fully understood, and studies have identified proteins in the family of TLR as important mediators of antigen presentation. ${ }^{24-26}$ Among these is TLR7, for which imiquimod is approved by the US Food and Drug Administration (FDA) for topical therapy of human papilloma virus, actinic keratosis and basal cell carcinoma, and has also been used to treat other skin diseases. Imiquimod has antiviral and antitumoral effects, ${ }^{24} 27-30$ and it can increase the magnitude and breadth of immune responses in patients. ${ }^{31} 32$ These studies indicate that imiquimod may be useful as an adjuvant for therapies requiring strong CTL immunity, such as tumor immunotherapy.

We report here the immunological and clinical findings from a clinical trial designed to obtain pilot data on the safety and immunogenicity of a peptide vaccine and granulocyte macrophage colony stimulating factor (GM-CSF) administered transdermally (via a skin patch) in incomplete Freund's adjuvant (IFA) or dimethyl sulfoxide (DMSO), with or without pretreatment of the skin with topical imiquimod. The study included vaccine injections after three transdermal vaccines, providing a positive control for each patient's ability to respond to vaccines. If effective, a transdermal vaccine delivery system may offer several advantages: (1) avoidance of chronic granulomatous changes and/or ulceration at injection sites, (2) the ability for patients to self-administer vaccines and (3) improved immunogenicity.

\section{METHODS}

\section{Participants}

Participants with resected American Joint Committee on Cancer (AJCC) stage II, III or IV melanoma, expressing HLA-A1, A2, or A3, were studied following informed consent, and with Institutional Review Board (IRB-HSR 11490) and FDA approval (BB-IND \# 11966). Inclusion criteria included: age 12 years or older, Eastern Cooperative Oncology Group (ECOG) performance status
$0-1$, adequate liver and renal function and completion of surgical therapy within the preceding 12 months. Exclusion criteria included: pregnancy; cytotoxic chemotherapy, interferon (IFN) or radiation administered within the preceding 4 weeks of randomization; known or suspected allergies to vaccine components; use of steroids; class III or IV heart disease; HIV or HCV seropositivity; systemic autoimmune disease with visceral involvement, or uncontrolled diabetes (Hemoglobin $\mathrm{A} 1 \mathrm{C} \geq 7 \%$ ).

Patients with stage IIB or III melanoma were eligible for this trial if they had previously failed IFN-alpha therapy, were medically ineligible for IFN, or if they chose not to take IFN after reviewing and demonstrating comprehension of a standardized approved information document about high-dose IFN developed at the University of Virginia.

\section{Clinical trial design}

This was an open-label, four-arm, randomized phase I study of a transdermal vaccine comprizing a mixture of twelve major histocompatibility complex (MHC) class I-restricted melanoma peptides (12MP), a tetanus helper peptide (tet) and GM-CSF. Participants were randomized to receive the $12 \mathrm{MP}+$ tet + GM-CSF vaccine transdermally (1) with or without imiquimod and (2) in an emulsion of IFA (Montanide ISA-51) or in a solution of DMSO. Randomization occurred in two stages and was based on random assignment with block sizes of 12, which resulted in cohorts of three participants per arm. Participants were randomized to one of four groups: (1) IFA; (2) IFA+ imiquimod; (3) DMSO; or (4) DMSO+ imiquimod. Beginning 3 weeks after the final transdermal vaccination (study week 5), participants received a series of booster vaccinations by traditional subcutaneous/intradermal injection.

The study was designed to assess safety (toxicity) and immunogenicity for each vaccine approach. Participants were randomly assigned to one of the four vaccine preparations. In order to monitor safety, randomization occurred in two stages. Accrual to treatment groups in the second stage was conditional on safety criteria being satisfied in the first stage. Toxicity was monitored continuously within each group, with stopping rules for unexpected treatment-related adverse events assessed after each stage of accrual. Total target accrual was set at a minimum of 24 eligible participants. Allowing for the unrealized possibility of withdrawals, total accrual to the study was 28 eligible participants. For this early phase trial, analyses were based on treatment received.

\section{Peptides used in vaccines}

The peptide vaccine used in all participants (12MP) includes peptides from melanocytic proteins (tyrosinase and gp100) and cancer-testis antigens (MAGE-A1, MAGEA3, MAGE-A10 and NY-ESO-1), restricted by HLA-A1, A2 or $\mathrm{A}^{33334}$ (online supplemental table 1). The tetanus peptide also used in all participants is AQYIKANSKFIGITEL. $^{35} 36$ 


\section{Other vaccine components}

GM-CSF (Leukine) was manufactured by Berlex (Seattle, Washington, USA) as a clinical grade reagent, and was purchased from the UVA hospital pharmacy. Montanide ISA-51 adjuvant was purchased from Seppic (Paris, France) in sealed glass vials $(3 \mathrm{~mL} /$ vial $)$. Montanide ISA-51 had been prepared with oleic acid from a bovine source ('animal' formulation) for 23 participants and from a vegetable source for 5 participants. ${ }^{37}$ DMSO (Cryoserv) was purchased from the manufacturer (Edwards Lifesciences Research Medical.; Irvine, California, USA). Imiquimod (Aldara; $5 \%$ cream, $0.25 \mathrm{~g} /$ packet) was manufactured by $3 \mathrm{M}$ (St. Paul, Minnesota, USA) as a clinical grade reagent and was purchased from the UVA hospital pharmacy.

\section{Transdermal vaccination}

Participants were vaccinated in an upper arm if intact draining nodes were present, and in an upper thigh if neither arm was available (eg, prior bilateral axillary node dissections). Prior to day 0 , the vaccination site was designated with a permanent marker outline of a $5 \times 6 \mathrm{~cm}$ area. To diminish the keratin layer and prepare skin for patch application, participants were instructed to shave a $9 \times 10 \mathrm{~cm}$ area at that site on the morning of days 0,7 and 14.

The evening prior to each patch application, participants in study groups 2 and 4 were instructed to wash the marked skin site with mild soap and water, then to apply one single-use packet of imiquimod ( $5 \%$ cream, $0.25 \mathrm{~g}$ ) topically. The area was to be washed again with soap and water 6-10 hours later.

For patch applications on days 1,8 and 15 , each vaccine contained $100 \mu \mathrm{g}$ of each peptide in 12MP, $200 \mu \mathrm{g}$ tetanus peptide and $110 \mu \mathrm{g}$ GM-CSF in $2 \mathrm{~mL}$ final volume. For groups 1 and 2, the peptides and GM-CSF were prepared in a 1:1 water-in-oil emulsion with IFA made using the two-syringe method, and stability of each emulsion was confirmed by the water drop test. For groups 3 and 4, the peptides and GM-CSF were prepared in $25 \%$ DMSO. The final $2 \mathrm{~mL}$ volume was dropped on the $4.4 \mathrm{~cm} \mathrm{x} 6.0 \mathrm{~cm}$ absorbent pad portion of an $8.8 \times 10.2 \mathrm{~cm}$ commercially available Tegaderm+Pad $(3 \mathrm{M})$ transparent dressing. The designated transdermal immunization site was swabbed with alcohol and allowed to dry just prior to application of the dressing/vaccine. The dressing remained in place for 6 days and was removed by the participant on the day prior to the next study visit.

\section{Management of participants}

On day 1, participants were admitted for 24 hours to the General Clinical Research Center for patch application and postpatch blood draws performed at 5, 15, $30 \mathrm{~min}$, then every $30 \mathrm{~min}$ to 6 hours, hourly to 10 hours, then every 2 hours until24 hours. Blood was drawn for immunological studies at all subsequent outpatient visits: days $8,15,22$, and weeks 5, 8, 11, 12, 14, 17, 20 and 23 .
Beginning at week 5, participants in all study groups were vaccinated by injection using the same mixture of 12MP and tetanus peptides, now suspended in an emulsion with Montanide ISA-51 and GM-CSF. Injections were administered half intradermally and half subcutaneously at the same skin location where the vaccine patches had previously been placed. A total of six vaccines were injected at weeks 5, 8, 11, 14, 17 and 20. Figure $1 \mathrm{~A}$ is a study schematic.

\section{Toxicity assessment and stopping rules}

Toxicities recorded by each participant using daily diaries were reviewed by interview with a study physician or nurse each week. Toxicities were graded in accordance with National Cancer Institute (NCI) Common Toxicity Criteria V.3.0. Unexpected treatment-related adverse events included grade 2 allergic reactions, grade 3 nonhematological/metabolic toxicities and grade 4 hematologic/metabolic toxicities. Vaccine site ulceration was considered grade 2 unless it required antibiotic therapy, surgical debridement, or narcotic management for pain. In those cases, it was considered grade 3 .

\section{Clinical follow-up}

Participants were evaluated by study clinicians on an outpatient basis prior to protocol entry, at the date of each vaccine, 1 week after the third vaccine, and at week 26 . Thereafter, they were followed for survival. Evaluations included interval history and physical examinations with evaluation for vitiligo, ocular and visual changes and any evidence of tumor progression. Staging studies (CT scan of chest/abdomen/pelvis, MRI or CT scan of the brain, bone scan) were performed prior to protocol entry, and follow-up cross-sectional imaging was performed typically at least once per year or more often at the clinician's discretion, taking into account the stage of disease.

\section{Cell lines and peptides used in vitro}

C1R-A1, C1R-A2 and C1R-A3 are human EBV-transformed B-cell lines that lack expression of Class I MHC molecules, except that they have been transfected with genes for human HLA-A1, HLA-A2 and HLA-A3, respectively (provided by P. Creswell). ${ }^{30} 38$ An irrelevant peptide from HIV gag protein (SLYNTVATL) ${ }^{30} 39$ was used as a negative control.

\section{ELlspot assay}

Peripheral blood was collected before vaccination, weekly after transdermal vaccination, then $1-3$ weeks after each injected vaccination. Peripheral blood mononuclear cells (PBMC) were isolated by Ficoll gradient centrifugation, then cryopreserved in 10\% DMSO, 90\% fetal bovine serum. Lymphocytes were assayed by IFN- $\gamma$ ELIspot 14 days after a single sensitization in vitro with $40 \mu \mathrm{g} /$ $\mathrm{mL}$ synthetic peptide (in vitro stimulated, IVS ELIspot) or directly ex vivo (Direct ELIspot). Samples from prevaccination (defined as the baseline sample) and representative samples after one or more vaccinations were evaluated simultaneously. ELIspot assay methods have 
(A)

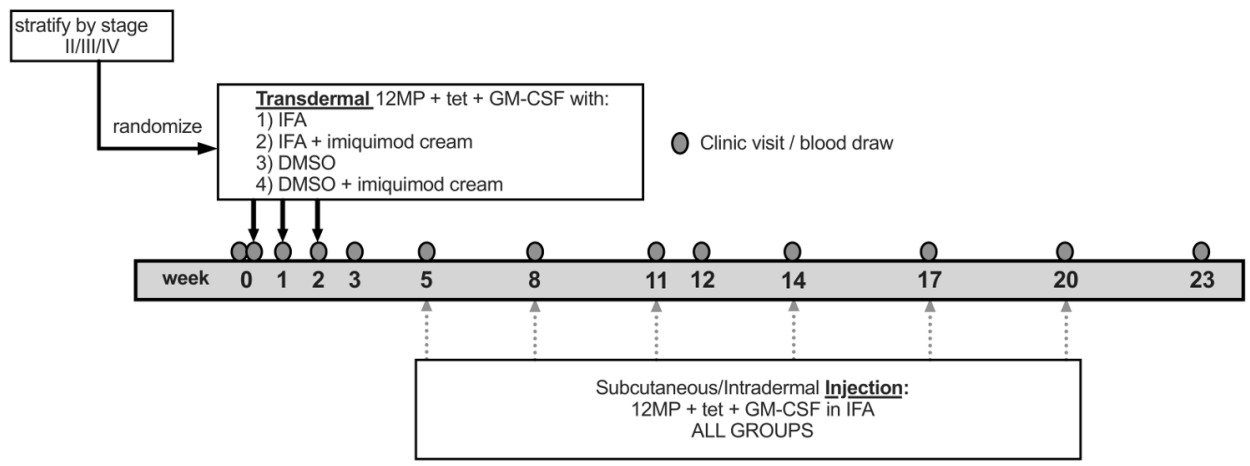

(B)

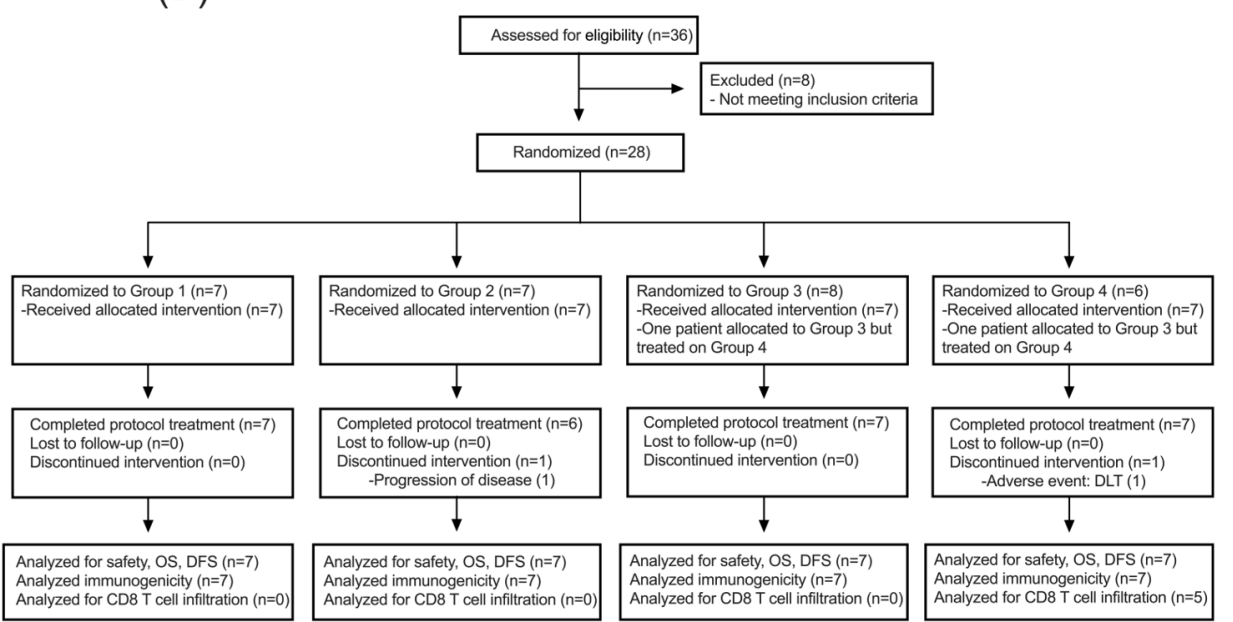

Figure 1 (A) Study protocol. Transdermal vaccination (at weeks 0,1,2) with 12MP + tet + GM-CSF with either IFA, IFA + imiquimod, or DMSO, DMSO + imiquimod. This was followed by subcutaneous/intradermal injection of 12MP + tet + GMCSF in IFA for all groups. Blood was drawn for analysis on weeks indicated by the gray oval. (B) Study diagram outlining the randomization, trial completion and analysis of enrolled patients. 12MP, $12 \mathrm{MHC}$ class I-restricted melanoma peptides; DFS, disease-free survival; DMSO, dimethyl sulfoxide; GM-CSF, granulocyte-macrophage colony-stimulating factor; IFA, Incomplete Freund's adjuvant; OS, overall survival; tet, tetanus helper peptide; DLT, dose-limiting toxicity.

been described. ${ }^{340}$ Each sample was tested in quadruplicate at several dilutions of lymphocytes.

Assessment of immunological response was based on the number of spots counted per 100000 cells plated and a calculated 'fold-increase' measure. Overall numbers of spots counted were scaled to account for the percentage of $\mathrm{CD} 4$ or $\mathrm{CD} 8$ cells present in each sample. To make comparisons between immune responses at different time points, the total number of spots $/ 100000$ for a given peptide was scaled to the negative control for each assay date. This ratio was then scaled to the baseline immune response in pretreatment PBMC. This was calculated as the 'fold-increase' $=\mathrm{N}_{\text {vax }} / \mathrm{N}_{\text {neg }}$ post-vaccination $\div \mathrm{N}_{\text {vax }} /$ $\mathrm{N}_{\text {neg }}$ at baseline, and used the following definitions: $\mathrm{N}_{\mathrm{vax}-}$ $=$ number of spots counted in response to stimulation with vaccine peptides and $\mathrm{N}_{\text {neg }}$ was the maximum count for each assay's two negative controls: C1R-A1, C1R-A2 or C1R-A3 tumor cell line either alone or pulsed with an irrelevant peptide. $\mathrm{R}_{\mathrm{vax}}=$ ratio of $\mathrm{N}_{\mathrm{vax}} / \mathrm{N}_{\text {neg }}$. When the pre-vaccine $R_{\text {vax }}$ value was zero, this value was converted to one, to avoid dividing by zero when calculating the fold-increase. For IVS ELISpot assays, a participant was considered to have a $\mathrm{T}$ cell response to vaccination only if all of the following criteria were met ${ }^{1}: \mathrm{N}_{\mathrm{vax}}$ exceeded $\mathrm{N}_{\text {neg }}$ by at least 100 cells per $100000 \mathrm{CD} 8^{+}$cells, ${ }^{{ }^{\mathrm{vax}}} \mathrm{R}_{\mathrm{vax}}>2,{ }^{3}\left(\mathrm{~N}_{\mathrm{vax}}{ }^{\mathrm{neg}}\right.$ $-1 \mathrm{SD}) \geq\left(\mathrm{N}_{\text {neg }}+1 \mathrm{SD}\right)$ and $^{4} \mathrm{R}_{\mathrm{vax}}$ postvaccination $\geq 2 \mathrm{x} \mathrm{R}_{\mathrm{vax}}$ prevaccine. For direct ELIspot assays of CD4 responses to tetanus, a participant was considered to have an immune response to vaccination if ${ }^{1} \mathrm{~N}_{\mathrm{vax}}$ exceeded $\mathrm{N}_{\text {neg }}$ by at least 20 cells per $100000 \mathrm{CD} 4^{+}$cells and the above criteria $2-4$ were met.

\section{Immunohistochemistry}

Immunohistochemistry was performed on a robotic platform (Ventana discover Ultra Staining Module, Ventana, Tucson, Arizona, USA). Tissue sections $(4 \mu \mathrm{m})$ were deparaffinized using EZ Prep solution (Ventana). A heatinduced antigen retrieval protocol set for $64 \mathrm{~min}$ was 
carried out using Cell Conditioner 1 (Ventana). Endogenous peroxidases were blocked with peroxidase inhibitor (CM1) for $8 \mathrm{~min}$ before incubating the section with CD8 antibody (Dako, Cat\#M7103) at 1:400 dilution for $60 \mathrm{~min}$ at room temperature. Antigen-antibody complex was then detected using DISC. OmniMap antimouse multimer RUO detection system and DISCOVERY ChromoMap DAB Kit (Ventana). All the slides were counterstained with hematoxylin and then dehydrated, cleared and mounted for assessment. The slides were then visually assessed by microscopy and the $\mathrm{CD} 8^{+} \mathrm{T}$-cell density was defined as the number of $\mathrm{CD}^{+}$cells/HPF present within the dermis and epidermis.

\section{Assay of systemic cytokines after patch application}

For evaluation of cytokines, blood was drawn from each participant at times $0,5 \mathrm{~min}, 60 \mathrm{~min}, 3$ hours, 6 hours, 12 hours and 24 hours after the first patch application. Serum was collected and stored at $-20^{\circ} \mathrm{C}$. Cytokine levels were measured by using a multiplex cytokine bead assay kit (Bio-Rad Laboratories, Hercules, California, USA) following the manufacturer's instructions. Briefly, sera from participants were thawed at $4^{\circ} \mathrm{C}$, diluted $1: 4$ in species-specific serum diluent and incubated at room temperature with pre-mixed beads in wells of a 96-well filter plate. The following analytes were included: interleukin 2 (IL2), IL4, IL5, IL7, IL10, IL12 (p70), IL13, IL15, GM-CSF, IFN-gamma and tumor necrosis factor (TNF)-alpha. After incubation, beads were washed three times using a vacuum plate manifold. Then, sequential incubations at room temperature with detection antibody for $30 \mathrm{~min}$ followed by streptavidin-PE for $10 \mathrm{~min}$ were done. After washing to remove unbound reactants, the plate was read using the Bio-Plex system in combination with Bio-Plex Manager software (Bio-Rad) to quantitate cytokine levels in serum.

\section{Statistical analysis}

The study was not designed to compare treatments groups but to provide initial assessment of toxicity and immune response. Therefore, frequency counts, descriptive statistics, graphical methods and point estimation with CIs around estimated parameters were the main methods of analysis. To determine the per cent of patients with an immune response during the transdermal vaccination period or injected vaccine period, the number of patients with an immune response during the period of interest was divided by the total number of patients with evaluable sample during that period and multiplied by 100 . Time to event distributions were estimated by the product-limit method of Kaplan-Meier.

\section{RESULTS \\ Participant groups}

A total of 36 individuals were consented, 28 (78\%) of whom eventually registered to the study. Reasons individuals did not register included screening failure $(n=5)$, withdrawn consent $(n=1)$, disease progression $(n=1)$, and other reasons $(\mathrm{n}=1)$.

Participant characteristics are summarized in table 1, and figure $1 \mathrm{~B}$ shows the randomization and analysis flow diagram. One participant was randomized to group 3 but inadvertently treated on group 4, thus all data for that participant were reported with group 4 results. Overall, there were seven participants treated in each study group. All were Caucasian, with one Hispanic participant (3.6\%). There were equal numbers of males and females. Median age was 51, similar among groups. ECOG performance

Table 1 Participant characteristics by treatment group

\begin{tabular}{lccccc}
\hline & Group 1 & Group 2 & Group 3 & Group 4 & Total \\
\hline N & 7 & 7 & 7 & 7 & 28 \\
Gender (female) & 4 & 5 & 3 & 2 & $14(50 \%)$ \\
Race-Caucasian & 7 & 7 & 7 & 7 & $28(100 \%)$ \\
Ethnicity-Hispanic & 0 & 0 & 1 & 0 & $1(4 \%)$ \\
Age-median (min-max) & $47(17-75)$ & $45(41-75)$ & $51(41-75)$ & $59(14-70)$ & $51(14-75)$ \\
ECOG Performance status & 7 & 6 & 7 & 7 & $27(96 \%)$ \\
Stage IIA & 0 & 1 & 0 & 3 & $4(14 \%)$ \\
Stage IIB & 0 & 0 & 0 & 1 & $1(4 \%)$ \\
Stage IIIA & 1 & 1 & 1 & 2 & $5(18 \%)$ \\
Stage IIIB & 0 & 3 & 4 & 0 & $7(25 \%)$ \\
Stage IIIC & 2 & 2 & 2 & 1 & $7(25 \%)$ \\
Stage IV & 4 & 0 & 0 & 0 & $4(14 \%)$ \\
HLA-A1 & 5 & 2 & 2 & 1 & $10(36 \%)$ \\
HLA-A2 & 4 & 5 & 5 & 6 & $20(71 \%)$ \\
HLA-A3 & 0 & 1 & 0 & 1 & $2(7 \%)$ \\
\hline
\end{tabular}


Table 2 Treatment-related toxicity by trial group

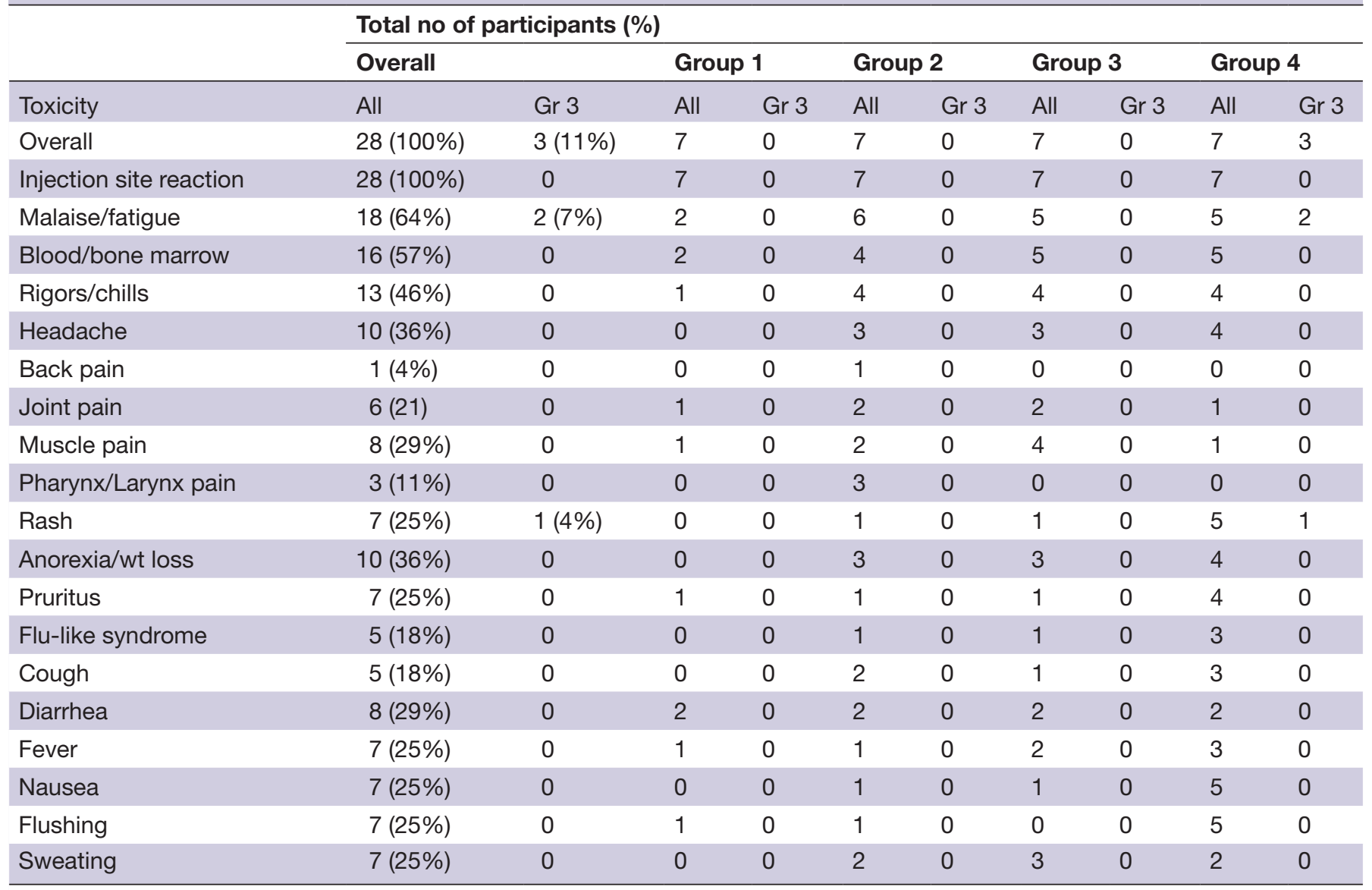

Gr, grade.

status was 0 in $96 \%$ of participants. AJCC stage at enrollment was II in $18 \%$, III in $68 \%$, and IV in $14 \%$, with higher stage IV participants in group 1 and lower stage participants in group 4. Participants expressed HLA-A1, A2, and A3, with A1 and A2 being most common. One participant was withdrawn early from study group 4 during transdermal vaccination because they experienced a severe vaccine-related rash, and one participant from group 2 discontinued treatment because of disease progression. All other participants completed the study protocol. All patients were evaluated for immune response through the treatment period.

\section{Safety}

Treatment-related adverse events experienced by at least $10 \%$ of participants are summarized in table 2 . There were no grade 4 toxicities or participant deaths during the study. Vaccine site reactions were common. Patch application was associated with grade 2 reactions in one participant (14\%) from groups 1 and 2 receiving topical vaccine in IFA, and with $4(57 \%)$ and $5(71 \%)$ participants in groups 3 and 4 , respectively, where vaccine was applied in DMSO. Subcutaneous/intradermal vaccine injection elicited grade 2 reactions in $93 \%$ of participants overall.
Prominent rashes were observed in five of seven participants in group 4 (DMSO + imiquimod), of which one was dose limiting. Rashes were widely distributed, not limited to the vaccination site. Dose-limiting symptoms occurred in a participant who had had extensive sun exposure during the first 2 weeks on study and developed a severe rash with distribution partially suggestive of photosensitivity (figure 2). Skin biopsy was consistent with a type IV hypersensitivity reaction. Other participants with rashes in group 4 went on to complete a full course of vaccination without recurrence of symptoms.

Non-specific constitutional symptoms listed in table 2 were very common and were largely grade 1 in severity. Less common treatment-related grade 1 toxicities (not listed in

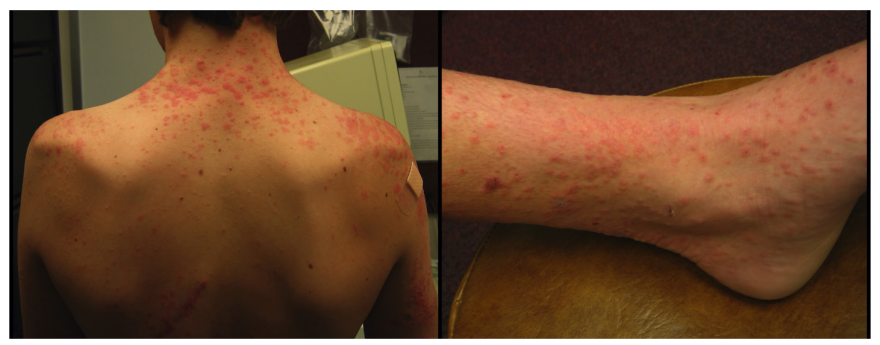

Figure 2 Dose-limiting rash in patient in group 4. 
(A)

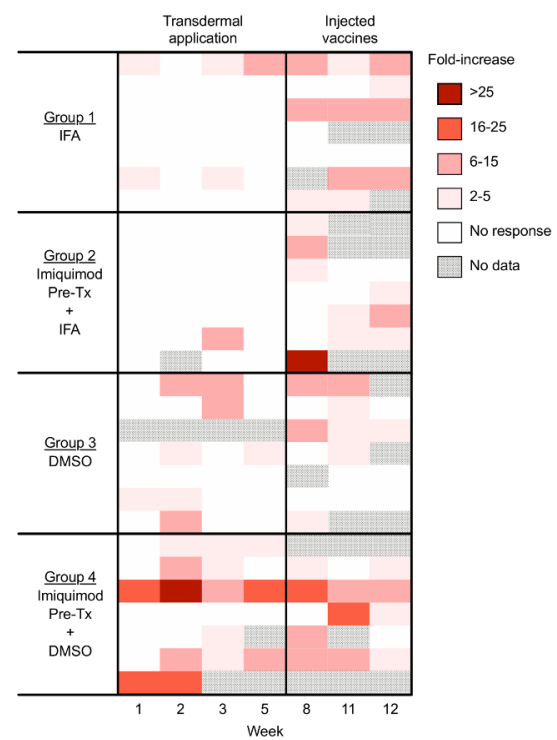

(B)

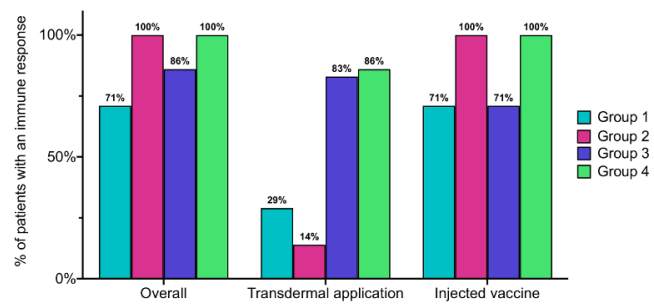

(C)

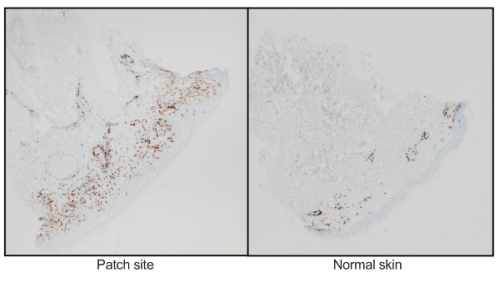

(D)

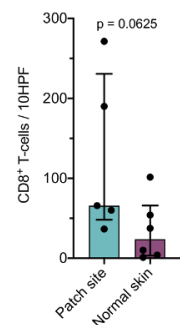

Figure $3 \mathrm{CD}^{+}$immune responses to class I MHC-restricted melanoma peptides (12MP) in in vitro stimulated (IVS) ELIspot assay. (A) Heatmap demonstrating the magnitude of immune response to transdermal and injected vaccines. Each patient is represented as a single row. Observed immune responses with fold increase over negative control less than $2 \mathrm{x}$ or those not meeting other criteria for response are shown in white. Gray represents unavailable data. (B) Percent of patients with an immune response to 12MP by group in the transdermal and injected time points. (C) Immunohistochemistry of a patch site biopsy and adjacent normal skin from one patient (group 4) stained with CD8 antibody demonstrating dense infiltrate of CD8 ${ }^{+}$cells compared with the normal skin. (D) Comparison of overall $C D 8^{+} T$ cell counts in patients from group four patch sites versus normal skin (median, IQR), with a trend toward higher $C D 8^{+} T$ cell count in the patch site; $p=0.0625$. Comparison was made using the Wilcoxon rank sum test with alpha=0.05. DMSO dimethylsulfoxide; HPF, high power field; IFA, incomplete Freund's adjuvant; Tx, treatment

table 2) were rhinitis $(n=3)$, nasal/paranasal reactions $(n=3)$, mucositis $(n=2)$, vomiting $(n=2)$, allergic reaction $(n=2)$, autoimmune reaction $(\mathrm{n}=1)$, hyperpigmentation $(\mathrm{n}=1)$, depression $(n=1)$, limb edema $(n=1)$, constipation $(n=1)$, and treatment-related bronchitis $(\mathrm{n}=1)$. The autoimmune reaction was an elevation in rheumatoid factor (44.9IU/ $\mathrm{mL}$ ) at week 20 and was asymptomatic.

Treatment-related metabolic toxicities included hyperglycemia in eight participants ( 7 grade 1,1 grade 2$)$, which is likely overestimated, as these were not typically based on fasting glucose levels. Other metabolic toxicities were uncommon and all grade 1: hyperkalemia $(n=2)$, hypokalemia $(n=2)$, hyponatremia $(n=2)$, hypoglycemia $(n=2)$, elevated creatinine $(n=2)$, and alkaline phosphatase $(n=1)$.

\section{Immunogenicity}

$\mathrm{CD}^{+}$T-cell responses to MHC class I-restricted peptides: IVS ELIspot assay

Overall, circulating immune responses to peptides were detected by IVS ELIspot in 52\% of participants during transdermal vaccination (weeks $1-5$ ) and in $85 \%$ of participants after traditional injected vaccine, during weeks $6-12$. Overall (weeks 1-12), 89\% of participants had a circulating immune response. The magnitude of immune responses to 12MP peptides detected by IVS ELIspot are shown in figure 3A.

Transdermal vaccines with IFA were infrequently immunogenic, with two of seven $(29 \%)$ responding in group 1, and one of seven (14\%) in group 2 (figure 3B).
Transdermal vaccines using DMSO were more frequently immunogenic, with 5 of 6 (83\%) participants in group 3 responding (one patient not evaluable during transcutaneous immunization) and six of seven (86\%) in group 4 (figure 3B). After initiation of injected vaccines (beyond week 5), responses were detected in $89 \%$ of participants: $5(71 \%), 7(100 \%), 5(71 \%)$ and $5(100 \%, 2$ patients not evaluable) of participants in groups 1-4, respectively (figure 3B). IVS and Direct ELIspot absolute values are shown in online supplemental table 2.

Among participants with $\mathrm{CD}^{+} \mathrm{T}$ cell responses to transdermal vaccination in weeks $1-5$, mean numbers of peptides eliciting responses were 1.5 (group 1), 2.0 (group 2), 1.0 (group 3) and 2.3 (group 4). In responders overall, the mean number of peptides eliciting responses were $2.0,1.6,1.5$, and 2.1 in groups $1-4$, respectively.

Direct ELIspot assays performed with 12MP peptides detected immune responses in only $19 \%$ of participants overall during transdermal vaccination $(2,2,0$, and 1 in groups $1-4$, respectively) and $16 \%$ in subsequent weeks $(0$, 2,1 and 0 in groups $1-4$, respectively, online supplemental table 3$)$.

Immunohistochemistry of the vaccine site and normal skin was performed for participants in the group with highest fraction of transdermal immune responses (group 4) in order to examine $\mathrm{CD}^{+} \mathrm{T}$ cell infiltration. The infiltrating $\mathrm{CD}^{+} \mathrm{T}$ cell density in the patch-site trended 
(A)

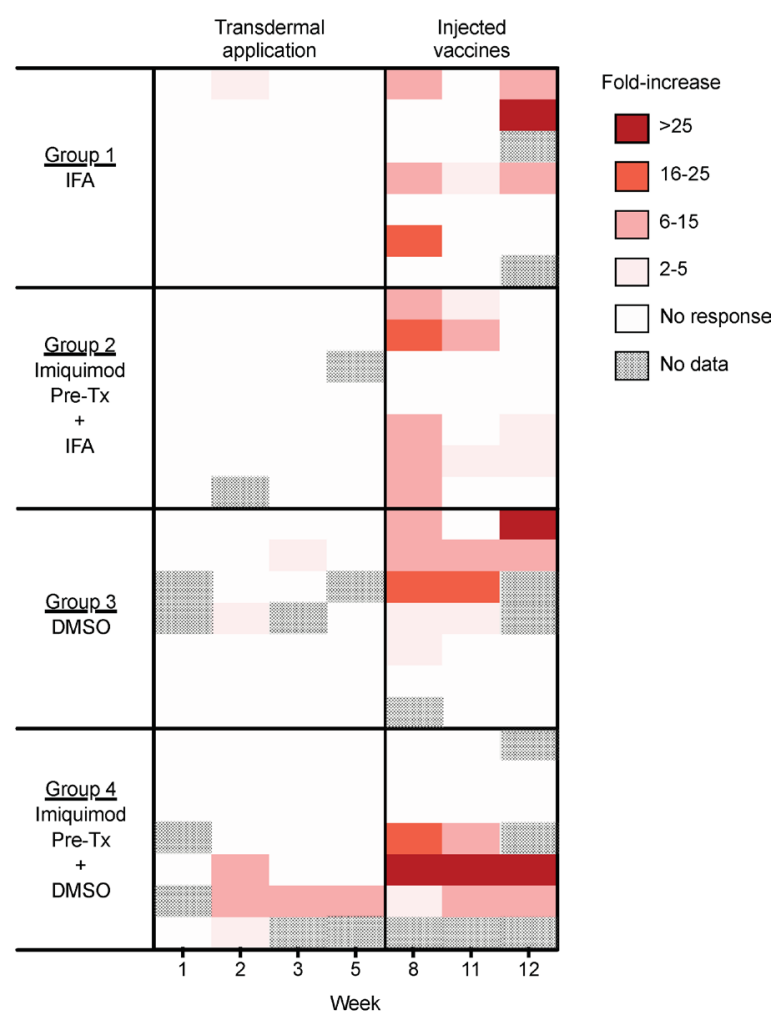

(B)

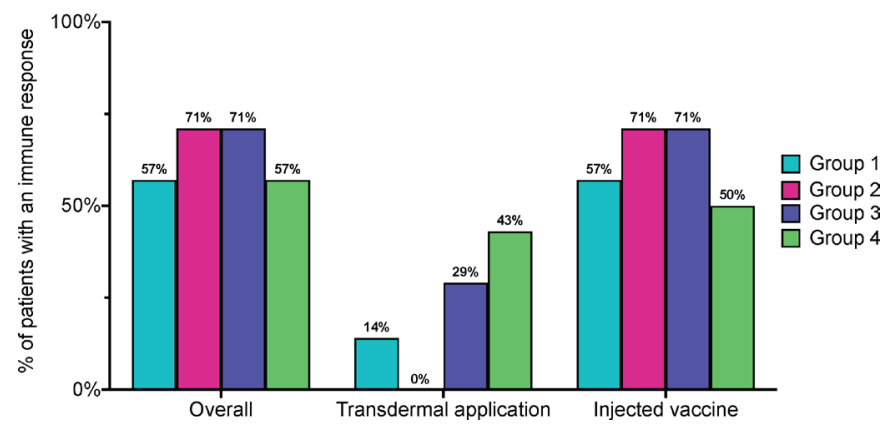

Figure $4 \mathrm{CD}^{+}$immune responses to class II MHCrestricted tetanus helper peptide in direct ELIspot assay. (A) Heatmap demonstrating the magnitude of immune response to transdermal and injected vaccines. Each patient is represented as a single row. Observed immune responses with fold increase over negative control less than $2 x$ or those not meeting other criteria for response are shown in white. Gray represents unavailable data. (B) Percent of patients with an immune response to tetanus helper peptide by group in the transdermal and injected time points. DMSO, dimethylsulfoxide; IFA, incomplete Freund's adjuvant; Tx, treatment.

higher than in normal skin $(\mathrm{p}=0.0625)$. Representative images and the comparison are shown in figure 3C,D.

$\mathrm{CD} 4^{+} \mathrm{T}$ cell responses to HLA-DR-restricted tetanus helper peptide: direct ELlspot assay

The magnitude of responses to tetanus helper peptide measured by direct ELIspot assays is shown in figure 4A. During transdermal vaccination, responses were detected in 1 of $7(14 \%), 0$ of 7,2 of $7(29 \%)$ and 3 of $7(43 \%)$ participants in groups 1-4, respectively, all with foldincreases less than 10. After starting vaccine injections on week $5,59 \%$ of participants had immune responses to tetanus peptide: $4(57 \%), 5(71 \%), 5(71 \%)$ and $3(50 \%$, 1 patient not evaluable during injected vaccine period) participants in groups 1-4, respectively. Direct ELIspot absolute values are shown in online supplemental table 4 .

\section{Impact on systemic cytokines}

To assess whether GM-CSF in the vaccines was increased in circulation after transdermal vaccination or whether the vaccines induced other inflammatory cytokines systemically, serum was evaluated by multiplex cytokine bead array at the following time points: time 0 (immediately before patch application), $5 \mathrm{~min}$, then at 1, 3, 6, 12 and 24 hours. In a cytokine panel including IL2, IL4, IL5, IL7, IL10, IL12(p70), IL13, IL15, GM-CSF, IFN-gamma and TNF-alpha, no change in circulating levels was observed in response to transdermal vaccination (data not shown).

\section{Clinical outcomes: 10-year survival}

Median follow-up time was 13.7 years (range 5.3 months to 14.5 years). Overall survival at 10 years was $66.7 \%$ (95\% CI $46 \%$ to $81 \%$ ) (figure 5A). Ten-year disease-free survival (DFS) was at $44.2 \%$ (95\% CI $25 \%$ to $62 \%$ ) with a median DFS of 7.14 years (figure 5B).

\section{DISCUSSION}

In this phase I trial of transdermal vaccination with $12 \mathrm{MP}+$ tet + GM-CSF in IFA or DMSO, with or without imiquimod, we evaluated toxicity and cellular immune responses. The study provided proof of principle that transdermal delivery of a peptide-based vaccine can induce melanoma-reactive $\mathrm{T}$ cells. Toxicities were limited to mild (grade 1/2) local and systemic reactions in the majority of participants and were acceptable in all but one participant. That participant recovered well after discontinuing vaccines, had a $\mathrm{T}$ cell response and has remained clinically free of disease.

Systemic cutaneous rash of consistent character was prominent in participants receiving imiquimod +DMSO. The presence of systemic rash in five of seven participants in this group suggested that DMSO may have resulted in circulatory absorption of imiquimod and increased systemic toxicity, despite a low dose of the drug. A broad range of systemic reactions to topical imiquimod have been reported elsewhere, including disseminated psoriatic lesions, lichenoid reactions, erosive pustular dermatoses and several others. ${ }^{41-43}$ Many of these reactions have a type IV hypersensitivity component, and biopsy of the dose-limiting rash in our study was consistent with this. In the participant with the dose-limiting reaction, the increased prominence of lesions in sun-exposed areas suggested a photosensitivity effect. Cutaneous inflammation from extensive sun exposure may have exacerbated a milder imiquimod reaction, as the drug does not typically 
(A)

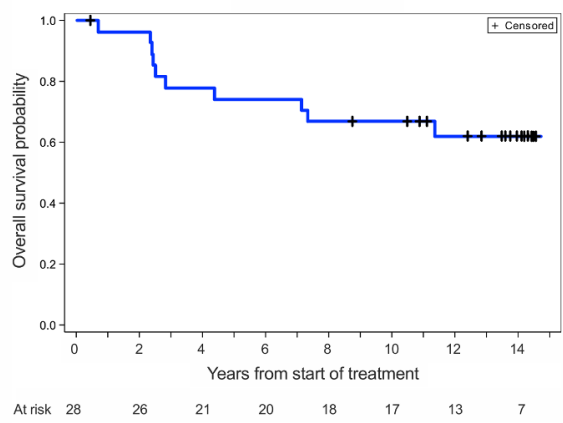

(B)

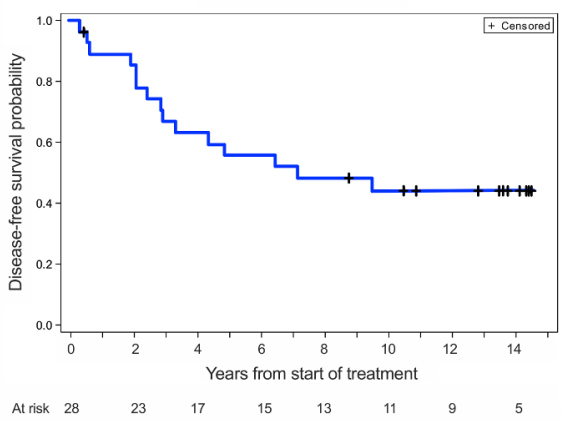

Figure 5 Kaplan-Meier overall survival (A) and disease-free survival (B) curves for all subjects.

react with normal, uninflamed skin. Since similar reactions were not observed in group 3, we attribute these toxicities to the combination and timing of imiquimod plus DMSO rather than to DMSO alone. It may also be safe with different doses or schedules of TLR agonists, or if patients are counseled to minimize sun exposure for several days after each vaccine.

Transdermal vaccination with peptides in DMSO yielded high rates of $\mathrm{CD}^{+} \mathrm{T}$ cell immune responses, $83 \%$ (group 3 ) and $86 \%$ (group 4), while responses to transdermal vaccination in Montanide ISA-51 were quite low: 29\% (group 1) and 14\% (group 2). This was not surprizing as IFA was unlikely to be a potent adjuvant across the dermal barrier, but could have served as a depot allowing for slow release of antigen through the skin. While this study was not designed to test persistence of immune responses to transdermal vaccination past week 5, in group 4, all but one who had a response during transdermal vaccination (at weeks $0,1,2$ ) still had a response persisting through week five or to the last evaluable time point.

Direct ELIspot assays detected few $\mathrm{CD} 8^{+} \mathrm{T}$ cell responses to melanoma peptides, which may be a result of GM-CSF. In a multicenter randomized controlled trial, we found that melanoma participants vaccinated with multipeptide vaccine and GM-CSF had significantly lower circulating T-cell response than those participants vaccinated without GM-CSF, and that detection of responses in direct ELISpot was dramatically decreased when GM-CSF was included in the adjuvant. ${ }^{44} 45$ Thus, DMSO appears to be useful for delivery of peptides in transdermal peptide vaccination. Vaccines without GM-CSF, but with other absorbable adjuvants may well be even more immunogenic.

The question of antigen-specific $\mathrm{CD} 4^{+} \mathrm{T}$ cell induction was addressed through direct ELIspot assays with tetanus helper peptide. There were only a few small magnitude $\mathrm{CD}^{+} \mathrm{T}$ cell immune responses to transdermal vaccination. After initiation of injected vaccines, groups 3 and 4 each had several participants with large-magnitude immune responses, suggesting a possible priming effect from transdermal immunization with DMSO. An observed priming effect with transdermal immunization may represent the establishment of a strong memory $\mathrm{T}$ cell response, with rapid $\mathrm{T}$ cell proliferation on restimulation.
The median DFS was 7.14 years and overall survival was $67 \%$ at 10 years, which is somewhat unusual for this highrisk group of participants ( $82 \%$ stage III-IV, table 1 ) using AJCC V.5 before the checkpoint blockade era. This may be due to the vaccines, or may reflect favorable patient selection in this small pilot study. For our study population, the median time from surgery to study enrollment was 5.2 months (5.2 months for survivors; 5.0 months for non-survivors). This interval may have excluded patients with rapidly progressing disease.

In this study, shaving of the patch site was performed in order to disrupt the stratum corneum prior to patch application. One of the challenges of transcutaneous vaccination is the barrier function of the skin. The skin's outermost layer, the stratum corneum, serves as a hydrophobic barrier which impedes penetration of topically applied vaccine. Numerous approaches have been developed in order to bypass the stratum corneum, the most common of which are shaving, skin stripping with tape, microneedles and nanoparticles. ${ }^{46-48}$ While microneedle and nanoparticle-based techniques have shown promise, shaving or tape stripping are appealing due to their effectiveness, simplicity, and low cost. Typical cancer vaccine regimens require repeated office visits which is one reason a simple, transcutaneous vaccine regimen is so appealing. Transcutaneous vaccination with either shaving or tape stripping of the patch site can be done at home by the patient without the need for specialized equipment other than the prescribed vaccine patch, a razor, or some tape.

In summary, we have demonstrated proof of principle that transdermal peptide vaccination can generate high rates of antigen-specific $\mathrm{CD} 8^{+} \mathrm{T}$ cell immune responses, with DMSO as a useful delivery agent for further study. Imiquimod may have value as an adjuvant, to increase the magnitude and breadth of immune responses. The increased systemic toxicity with imiquimod and DMSO warrants caution in their future use together, but this may be manageable with added sun protection since photosensitivity may have contributed to the rashes. Transdermal vaccination with peptides plus DMSO may have a priming effect on $\mathrm{CD} 4^{+} \mathrm{T}$ cell response to subsequent injected vaccines. Transdermal priming for subcutaneously/intradermally injected vaccination may prove 
useful with further investigation and possibly in combination with checkpoint blockade therapies. Methods and adjuvants for transdermal peptide immunization have yet to be optimized, and these data encourage continued efforts toward this end.

Acknowledgements We greatly appreciate the work of Patrice Neese, Carmel Nail and Priscilla Merrill in care of the participants on this trial including vaccine administration, Cheryl F.Murphy, Nadejda V. Galeassi, Elizabeth Coleman in immune analyses and data management, and Robyn J. Fink, Beverely Turner, Jennifer Hodges, Kristy Scott and Alison Gaucher in coordination of the clinical research and follow-up.

Contributors All authors contributed to the manuscript writing. MOM, EPS, KTL and CLS completed the first draft of the manuscript. GRP and MS provided the statistical analyses and data management. EW, KAC-B, WCO, and DD, JWP, WWG and CLS assisted with clinical trial design, implementation, patient treatment and laboratory analyses.

Funding This study was supported by NIH R01 CA057653 and research training grants from the National Institutes of Health T32HL007849, T32CA163177 and 5T32CA163177-09. Support was also provided by the University of Virginia Cancer Center Support Grant (NIH/NCI P30 CA44579: Clinical Trials Office, Biorepository and Tissue Research Facility, Flow Cytometry Core, and Biomolecular Core Facility) and the UVA General Clinical Research Center (NIH M01 RR00847). Peptides used in this vaccine were prepared with philanthropic support from the Commonwealth Foundation for Cancer Research and Alice and Bill Goodwin. Additional philanthropic support was provided by the James and Rebecca Craig Foundation, George S. Suddock, Richard and Sherry Sharp, and the Rebecca Clary Harris Memorial Fellowship. No corporate funding support was provided for this study.

Disclaimer The content is solely the responsibility of the authors and does not necessarily represent the official views of the National Heart, Lung, and Blood Institute or the National Institutes of Health.

Competing interests Several of the peptides used in the peptide vaccine reported in this manuscript were discovered at the University of Virginia, and have been licensed through the UVA Licensing and Ventures group; CLS is an inventor for these peptides and receives a portion of the licensing payments.

Patient consent for publication Not required.

Provenance and peer review Not commissioned; externally peer reviewed.

Data availability statement Data are available on reasonable request.

Supplemental material This content has been supplied by the author(s). It has not been vetted by BMJ Publishing Group Limited (BMJ) and may not have been peer-reviewed. Any opinions or recommendations discussed are solely those of the author(s) and are not endorsed by BMJ. BMJ disclaims all liability and responsibility arising from any reliance placed on the content. Where the content includes any translated material, BMJ does not warrant the accuracy and reliability of the translations (including but not limited to local regulations, clinical guidelines, terminology, drug names and drug dosages), and is not responsible for any error and/or omissions arising from translation and adaptation or otherwise.

Open access This is an open access article distributed in accordance with the Creative Commons Attribution Non Commercial (CC BY-NC 4.0) license, which permits others to distribute, remix, adapt, build upon this work non-commercially, and license their derivative works on different terms, provided the original work is properly cited, appropriate credit is given, any changes made indicated, and the use is non-commercial. See http://creativecommons.org/licenses/by-nc/4.0/.

\section{ORCID iDs}

Max 0 Meneveau http://orcid.org/0000-0002-8410-061X

Craig L Slingluff http://orcid.org/0000-0002-6664-4373

\section{REFERENCES}

1 Timmerman JM, Czerwinski DK, Davis TA, et al. Idiotype-pulsed dendritic cell vaccination for B-cell lymphoma: clinical and immune responses in 35 patients. Blood 2002;99:1517-26.

2 Nagayama H, Sato K, Morishita M, et al. Results of a phase I clinical study using autologous tumour lysate-pulsed monocyte-derived mature dendritic cell vaccinations for stage IV malignant melanoma patients combined with low dose interleukin-2. Melanoma Res 2003;13:521-30.

3 Slingluff CL, Petroni GR, Chianese-Bullock KA, et al. Immunologic and clinical outcomes of a randomized phase II trial of two multipeptide vaccines for melanoma in the adjuvant setting. Clin Cancer Res 2007;13:6386-95.

4 O'Neill DW, Adams S, Goldberg JD, et al. Comparison of the immunogenicity of Montanide Isa 51 adjuvant and cytokine-matured dendritic cells in a randomized controlled clinical trial of melanoma vaccines. JCO 2009;27:3002.

5 Dillman RO, Cornforth AN, Nistor Gl, et al. Randomized phase II trial of autologous dendritic cell vaccines versus autologous tumor cell vaccines in metastatic melanoma: 5-year follow up and additional analyses. J Immunother Cancer 2018;6:19.

6 Slingluff CL, Petroni GR, Yamshchikov GV, et al. Clinical and immunologic results of a randomized phase II trial of vaccination using four melanoma peptides either administered in granulocytemacrophage colony-stimulating factor in adjuvant or pulsed on dendritic cells. JCO 2003;21:4016-26.

7 Butterfield LH. Dendritic cells in cancer immunotherapy clinical trials: are we making progress? Front Immunol 2013;4:454.

8 Anguille S, Smits EL, Lion E, et al. Clinical use of dendritic cells for cancer therapy. Lancet Oncol 2014;15:e257-67.

9 Slingluff CL, Petroni GR, Yamshchikov GV, et al. Clinical and immunologic results of a randomized phase II trial of vaccination using four melanoma peptides either administered in granulocytemacrophage colony-stimulating factor in adjuvant or pulsed on dendritic cells. J Clin Oncol 2003;21:4016-26.

10 Brinckerhoff LH, Kalashnikov VV, Thompson LW, et al. Terminal modifications inhibit proteolytic degradation of an immunogenic MART-1(27-35) peptide: implications for peptide vaccines. Int $J$ Cancer 1999;83:326-34.

11 Blanchet JS, Valmori D, Dufau I, et al. A new generation of Melan-AV MART-1 peptides that fulfill both increased immunogenicity and high resistance to biodegradation: implication for molecular antimelanoma immunotherapy. J Immunol 2001;167:5852-61.

12 Kalluri $\mathrm{H}$, Banga AK. Transdermal delivery of proteins. AAPS PharmSciTech 2011;12:431-41.

13 Glenn GM, Taylor DN, Li X, et al. Transcutaneous immunization: a human vaccine delivery strategy using a patch. Nat Med 2000;6:1403-6.

14 Partidos CD, Beignon A-S, Brown F, et al. Applying peptide antigens onto bare skin: induction of humoral and cellular immune responses and potential for vaccination. J Control Release 2002;85:27-34.

15 Bialojan A, Sohl J, Rausch J, et al. Transcutaneous immunization with CD40 ligation boosts cytotoxic T lymphocyte mediated antitumor immunity independent of CD4 helper cells in mice. Eur J Immunol 2019;49:2083-94.

16 Kozaka S, Tahara Y, Wakabayashi R, et al. Transcutaneous cancer vaccine using a reverse micellar antigen carrier. Mol Pharm 2020;17:645-55.

17 Lopez PA, Denny M, Hartmann A-K, et al. Transcutaneous immunization with a novel imiquimod nanoemulsion induces superior T cell responses and virus protection. J Dermatol Sci 2017;87:252-9.

18 Beignon AS, Briand JP, Muller S, et al. Immunization onto bare skin with heat-labile enterotoxin of Escherichia coli enhances immune responses to coadministered protein and peptide antigens and protects mice against lethal toxin challenge. Immunology 2001;102:344-51.

19 Seo N, Tokura Y, Nishijima T, et al. Percutaneous peptide immunization via corneum barrier-disrupted murine skin for experimental tumor immunoprophylaxis. Proc Natl Acad Sci U S A 2000;97:371-6.

20 Rausch J, Lopez PA, Bialojan A, et al. Combined immunotherapy: CTLA-4 blockade potentiates anti-tumor response induced by transcutaneous immunization. J Dermatol Sci 2017;87:300-6.

21 Glenn GM, Scharton-Kersten T, Vassell R, et al. Transcutaneous immunization with cholera toxin protects mice against lethal mucosal toxin challenge. J Immunol 1998;161:3211-4.

22 Itoh T, Celis E. Transcutaneous immunization with cytotoxic T-cell peptide epitopes provides effective antitumor immunity in mice. $J$ Immunother 2005;28:430-7.

23 Yagi $\mathrm{H}$, Hashizume $\mathrm{H}$, Horibe $\mathrm{T}$, et al. Induction of therapeutically relevant cytotoxic T lymphocytes in humans by percutaneous peptide immunization. Cancer Res 2006;66:10136-44.

24 Loré K, Betts MR, Brenchley JM, et al. Toll-Like receptor ligands modulate dendritic cells to augment cytomegalovirus- and HIV-1specific T cell responses. J Immunol 2003;171:4320-8.

25 Nurkkala M, Nordström I, Telemo E, et al. Mhc expression and chemokine production in the murine vagina following intra-vaginal 
administration of ligands to Toll-like receptors 3, 7 and 9. J Reprod Immunol 2007;73:148-57.

$26 \mathrm{Hemmi} \mathrm{H,} \mathrm{Akira} \mathrm{S.} \mathrm{TIr} \mathrm{signalling} \mathrm{and} \mathrm{the} \mathrm{function} \mathrm{of} \mathrm{dendritic} \mathrm{cells.}$ Chem Immunol Allergy 2005;86:120-35.

27 Burns RP, Ferbel B, Tomai M, et al. The imidazoquinolines, imiquimod and R-848, induce functional, but not phenotypic, maturation of human epidermal Langerhans' cells. Clin Immunol 2000;94:13-23.

28 Thomsen LL, Topley P, Daly MG, et al. Imiquimod and resiquimod in a mouse model: adjuvants for DNA vaccination by particle-mediated immunotherapeutic delivery. Vaccine 2004;22:1799-809.

29 Huang SJ, Hijnen D, Murphy GF, et al. Imiquimod enhances IFN-gamma production and effector function of T cells infiltrating human squamous cell carcinomas of the skin. $J$ Invest Dermatol 2009;129:2676-85.

30 Daayana S, Elkord E, Winters U, et al. Phase II trial of imiquimod and HPV therapeutic vaccination in patients with vulval intraepithelial neoplasia. Br J Cancer 2010;102:1129-36.

31 Todd RW, Steele JC, Etherington I, et al. Detection of CD8+ T cell responses to human papillomavirus type 16 antigens in women using imiquimod as a treatment for high-grade vulval intraepithelial neoplasia. Gynecol Oncol 2004;92:167-74.

32 Mauldin IS, Wages NA, Stowman AM, et al. Topical treatment of melanoma metastases with imiquimod, plus administration of a cancer vaccine, promotes immune signatures in the metastases. Cancer Immunol Immunother 2016;65:1201-12.

33 Kittlesen DJ, Thompson LW, Gulden PH. Human melanoma patients recognize an HLA-A1-restricted CTL epitope from tyrosinase containing two cysteine residues: implications for tumor vaccine development [published erratum appears in J Immunol 1999 Mar 1;162(5):3106]. J Immunol 1998;160:2099-106.

34 Parkhurst MR, Salgaller ML, Southwood S, et al. Improved induction of melanoma-reactive CTL with peptides from the melanoma antigen gp100 modified at HLA-A*0201-binding residues. J Immunol 1996;157:2539-48

35 Slingluff CL, Yamshchikov G, Neese P, et al. Phase I trial of a melanoma vaccine with gp100(280-288) peptide and tetanus helper peptide in adjuvant: immunologic and clinical outcomes. Clin Cancer Res 2001;7:3012-24.

36 Slingluff CL, Engelhard VH, Ferrone S. Peptide and dendritic cell vaccines. Clin Cancer Res 2006;12:2342s-5.
37 Slingluff CL, Petroni GR, Smolkin ME, et al. Immunogenicity for CD8+ and CD4+ T cells of 2 formulations of an incomplete Freund's adjuvant for multipeptide melanoma vaccines. $J$ Immunother 2010;33:630-8.

38 Storkus WJ, Salter RD, Cresswell P, et al. Peptide-Induced modulation of target cell sensitivity to natural killing. J Immunol 1992;149:1185-90.

39 Johnson RP, Trocha A, Yang L, et al. Hiv-1 GAG-specific cytotoxic T lymphocytes recognize multiple highly conserved epitopes. fine specificity of the GAG-specific response defined by using unstimulated peripheral blood mononuclear cells and cloned effector cells. J Immunol 1991:147:1512-21.

40 Chianese-Bullock KA, Pressley J, Garbee C, et al. MAGE-A1-, MAGE-A10-, and gp100-derived peptides are immunogenic when combined with granulocyte-macrophage colony-stimulating factor and montanide ISA-51 adjuvant and administered as part of a multipeptide vaccine for melanoma. J Immunol 2005;174:3080-6.

41 Chan MP, Zimarowski MJ. Lupus erythematosus-like reaction in imiquimod-treated skin: a report of 2 cases. Am J Dermatopathol 2011;33:523-7.

42 O'Mahony C, Yesudian PD, Stanley M. Imiquimod use in the genital area and development of lichen sclerosus and lichen planus. Int $J$ STD AIDS 2010;21:219-21.

43 Greenberg HL, Cohen JL, Rosen T, et al. Severe reaction to 5\% imiquimod cream with excellent clinical and cosmetic outcomes. $J$ Drugs Dermatol 2007:6:452-8.

44 Faries MB, Hsueh EC, Ye X, et al. Effect of granulocyte/macrophage colony-stimulating factor on vaccination with an allogeneic wholecell melanoma vaccine. Clin Cancer Res 2009;15:7029-35.

45 Slingluff CL, Petroni GR, Olson WC, et al. Effect of granulocyte/ macrophage colony-stimulating factor on circulating CD8+ and CD4+ T-cell responses to a multipeptide melanoma vaccine: outcome of a multicenter randomized trial. Clin Cancer Res 2009;15:7036-44.

46 Choi MJ, Zhai H, Löffler H, et al. Effect of tape stripping on percutaneous penetration and topical vaccination. Exogenous Dermatology 2003;2:262-9.

47 Kim Y-C, Park J-H, Prausnitz MR. Microneedles for drug and vaccine delivery. Adv Drug Deliv Rev 2012;64:1547-68.

48 Toyoda M, Hama S, Ikeda Y, et al. Anti-Cancer vaccination by transdermal delivery of antigen peptide-loaded nanogels via iontophoresis. Int J Pharm 2015;483:110-4. 